\title{
Visualization of blood injection into the epidural space during the blood patch for the patient with intracranial hypotension
}

\author{
Yasuhiro Morimoto, MD \\ Department of Anesthesia, Ube Industries Central Hospital \\ 750 Nishikiwa Ube Yamaguchi JAPAN \\ yasumorimo@gmail.com
}

(Introduciton)

An epidural blood patch is used for the treatment of intracranial hypotension, as well as for the treatment of post-dural puncture headache. Fluoroscopy is commonly used because correct injection into the epidural space is essential. Recently, use of ultrasound has been suggested as an alternative to fluoroscopy, in order to avoid the risk of radiation exposure. Herein, we describe our technique.

\section{(Case)}

The patient was 57 years old male, who was diagnosed with spontaneous intracranial hypotension in our hospital. Preoperative radionuclide cisternography indicated a cerebrospinal fluid leak in the upper part of the lumbar spine. Therefore, an epidural blood patch was planned at the level of $L 3 / 4$. The procedure was performed in the lateral decubitus position. Ultrasound observation by curved array probe (Sonosite SII, Fujifilm Medical Japan, Tokyo, Japan) was used to confirm the needle insertion level and to measure the skin-to-dura distance. The probe was placed $2 \mathrm{~cm}$ lateral to the spinous process. A slight medial tilt was used to visualize the spine in a paramedian sagittal oblique view, in order to evaluate the sagittal spread of blood. On the basis of ultrasound observations, a 18G Tuohy needle was inserted at the level of L3/4 by the median approach, without ultrasound. After the needle had reached the epidural space, a catheter was inserted $3 \mathrm{~cm}$ into the epidural space.
The patient's blood, combined with a small amount of air (less than $0.2 \mathrm{~mL}$ ), was injected through the catheter with ultrasound observation (Video). A hyperechoic flash was observed in both anterior and posterior epidural spaces at the level of L2/3 after the injection of blood (Figure). Enlargement of the posterior epidural space was also observed. Twenty milliliters of blood were injected without any neurologic symptoms.

(Conclusion)

In this case, we could visualize blood injection in the posterior and anterior epidural spaces by real-time ultrasound observation through the addition of a small volume of air in the blood. Previously, we injected the blood without air, but could not observe the injection image; thus, the small volume of air facilitated the visualization of blood in the epidural space. An evaluation of injection volume and the cranial spread of blood in the epidural space should be considered in future cases.
Before injection

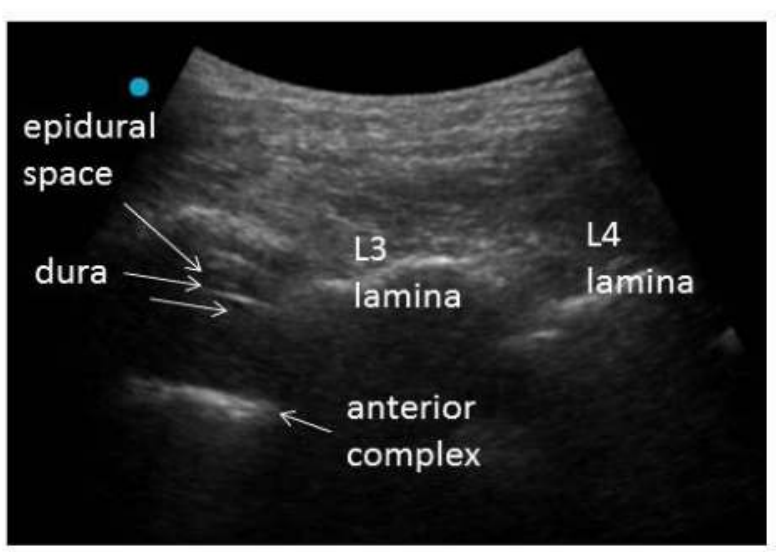

After injection

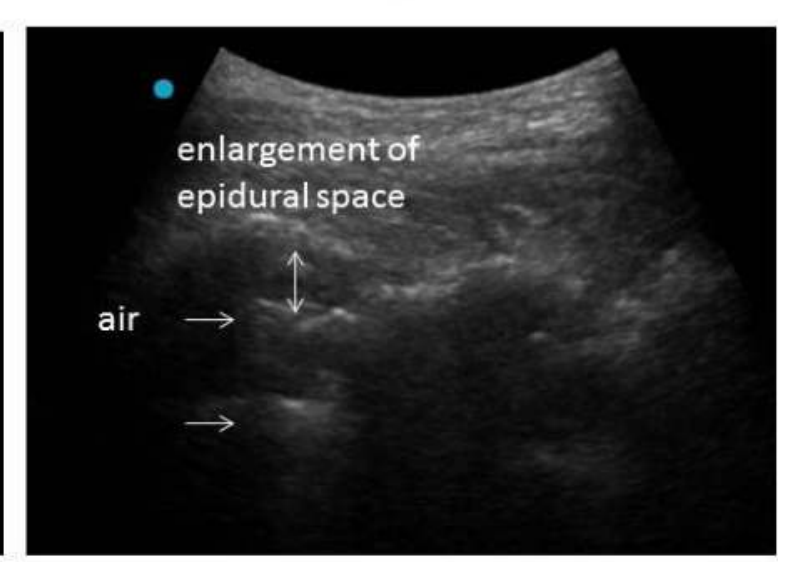

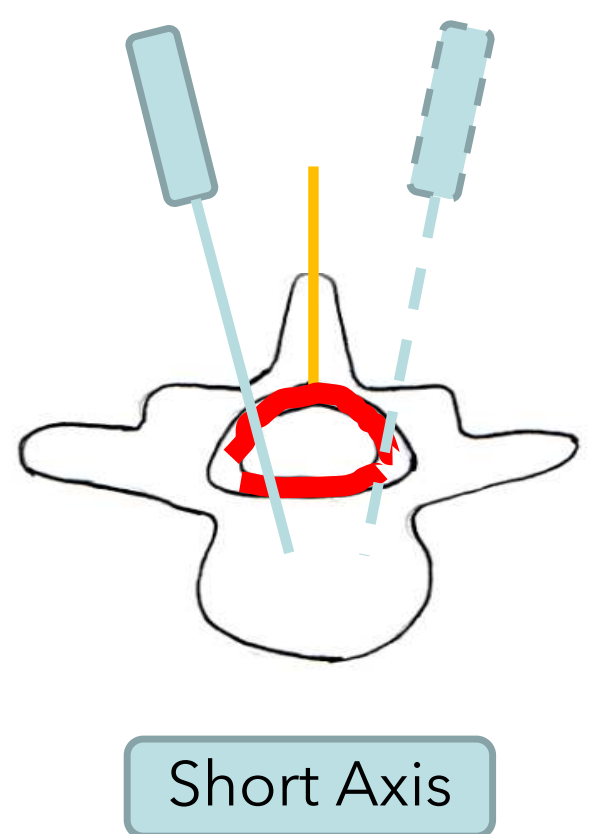

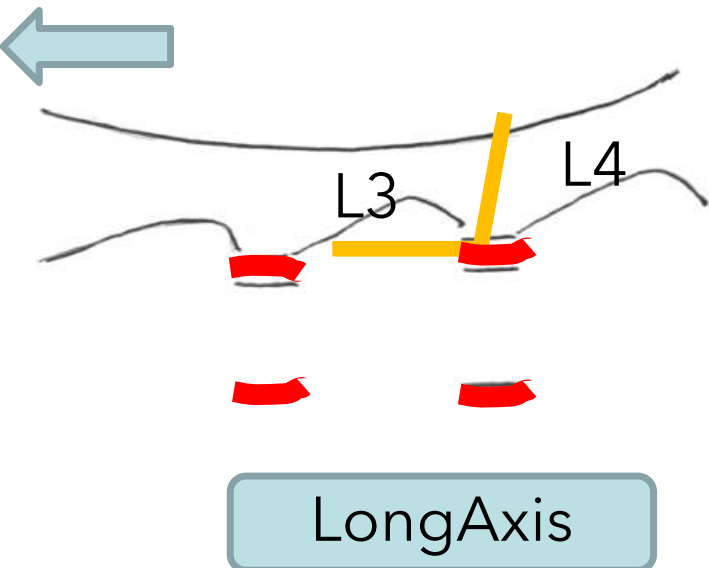

